\title{
Minding the Gap(s): Hospitalists Experience Aspirational, Safety, and Knowledge Deficits That Prevent Them From Practicing POCUS
}

\author{
Stephanie Conner, MD¹; David Chia, MD, MSc²; Farhan Lalani, MD'; Meghan O’Brien, MD, MBE²; James Anstey, \\ $\mathrm{MD}^{1}$; Nima Afshar, $\mathrm{MD}^{3}$; Trevor Jensen, MD, MSc ${ }^{1}$
}

(1) UCSF Helen Diller Medical Center at Parnassus Heights

(2) Zuckerberg San Francisco General Hospital and Trauma Center

(3) San Francisco VA Medical Center

\begin{abstract}
Point-of-care ultrasound (POCUS) has been a mainstay of clinical decision-making in the intensive care unit and emergency department for more than a decade, but adoption into hospital medicine has lagged behind. Recently, internal medicine residency programs have started to develop POCUS curricula for trainees, though concurrent hospitalist training programs have been limited

to date, with little consensus on what hospitalist-oriented curricula should entail. As such, there is wide variability amongst hospitalists with respect to utilization of, training in, and proficiency in POCUS. We conducted a two-part survey of internal medicine hospitalists at our institution: (1) needs assessment that focused on prior training, attitudes and perspectives, current practices, desired use, and barriers to clinical integration; and (2) knowledge test of exam indications, image interpretation, medical decisionmaking, and understanding of limitations. Our results demonstrate that a majority of hospitalists felt that POCUS was important for diagnostic purposes and that they would benefit from POCUS-specific education. Inadequate training was the most cited barrier to POCUS use. Hospitalist knowledge was lacking in all domains, particularly image interpretation and clinical integration. As a result, we created a three-tiered training program meant to engage: (1) All hospitalists in basic knowledge and appropriate use of POCUS,

(2) Some hospitalists in hands-on skill acquisition and image interpretation, and (3) Few hospitalists in mastery of POCUS with resultant formal credentialing. A tiered approach to POCUS training for hospitalists ensures a fundamental cognitive understanding of POCUS for all, but also facilitates hands-on training for those who are committed to further skillset development.
\end{abstract}

\section{Background}

Point-of-care ultrasound (POCUS) is increasingly recognized as an important real-time diagnostic tool in hospital medicine for a variety of clinical indications, including dyspnea, hypotension, volume assessment, skin and soft tissue infections, and others [1-8]. The incorporation of POCUS into clinical decision-making has been shown to increase the speed and accuracy of initial diagnosis, decrease procedural complications, and increase patient satisfaction compared to usual care [911]. While POCUS has been adopted and studied within the emergency department and intensive care unit for nearly two decades, its integration into internal medicine practice has lagged behind.

In recent years, POCUS training for hospitalists has gained momentum. The American Medical Association (AMA) has stated that "ultrasound imaging is within the scope of practice of appropriately trained physicians," and the American College of Physicians (ACP) has announced that it "will collaborate with other professional societies to facilitate the implementation of appropriate uses of POCUS throughout internal medicine training and practice." [12, 13]. In 2018, the Society of Hospital Medicine (SHM) released a position statement defining elements of cardiac, pulmonary, abdominal, musculoskeletal, vascular, and procedural ultrasound as within the scope of the POCUS-trained hospitalist [14]. Additionally, there is increasing proliferation of institutionally supported faculty and residency curricula across academic medical centers [15-17].

Despite support from national societies and expansion of POCUS training programs, no consensus exists for what components a POCUS curriculum should include. Ma et al. proposed that the minimum requirements for a 3-year internal medicine residency program curriculum, based on conclusions from an expert consensus method, should consist of: inferior vena cava, lung $b$-lines, pleural effusion, abdominal free fluid, central venous catheterization, thoracentesis, and paracentesis [18]. Mathews et al. modeled a hospitalist-oriented curriculum after the American College of Chest Physicians (ACCP) Certificate of Completion (COC) program that included broader domains: machine controls ("knobology"), vascular, lung, abdominal, and cardiac ultrasound [19]. Alternatively, Bahner et al. have argued that while programs may need to vary in content in order to be specialty-specific, there should be a standard educational and clinical practice model that all programs follow: the IAIM model [20]. This model focuses the learner on four domains for any POCUS application: Indication, Acquisition, Interpretation, and Medical Decision-Making. By applying this educational framework to a POCUS 
curriculum, physicians are guided towards appropriate educational and clinical use of any application.

We hypothesize that there has been wide variability of hospitalist exposure to POCUS training, leading to high variance with respect to competence, knowledge, and utilization of this skillset. Here, we describe the results of a two-part survey performed within our institution's three academic medical centers in an effort to better describe the current culture of POCUS understanding and education within hospital medicine.

\section{Methods}

We conducted a two-part survey in order to better understand present POCUS knowledge and practice amongst internal medicine hospitalists in order to create an educational program that is effective, accessible, and robust.

First, we created and disseminated a needs assessment within the Divisions of Hospital Medicine in three large, urban, academic hospitals. It involved a 13-item, webbased questionnaire focused on attitudes and perspectives, prior training, current practices and competencies, barriers to clinical integration, and aspirational goals.

Second, we conducted a web-based knowledge test to hospitalists within one of the three divisions. This test was comprised of 15 multiple choice questions that assessed different elements of the I-AIM model for a number of core POCUS applications: cardiac, IVC, lung, renal/ bladder, skin and soft tissue ultrasound. Responses to the knowledge test were grouped by level of experience as a hospitalist (0-3 years, 4-6 years, 7-10 years, >10 years). Statistical analysis was performed using analysis of variance (ANOVA).

Both parts of the survey were reviewed by the institutional IRB and granted exemption status prior to dissemination.

\section{Results}

\section{Needs Assessment}

The response rate for the needs assessment was $64 \%$ (82/129). Most hospitalists had little formal training in POCUS with approximately four fifths of hospitalists reporting fewer than ten hours of training with respect to dedicated didactics $(81 \%)$, supervised practice $(85 \%)$ and image interpretation (84\%). Key findings from this survey are summarized in Table 1.

Despite their limited experience with POCUS, the vast majority of hospitalists felt that it was important for diagnostic purposes in internal medicine (93\%) and that it should be a formal part of residency training (88\%). Furthermore, almost all hospitalists $(93 \%)$ believed that they would benefit from a dedicated POCUS training
Table 1. Key Findings of the Hospitalist Needs Assessment

\section{Importance:}

I believe POCUS is important for diagnostic purposes in internal medicine.

$93 \%$

I believe POCUS should be a formal part of residency training.

$88 \%$

\section{Desired Use:}

Given proper training and access to equipment, I foresee myself using POCUS regularly to assist with $\quad 73 \%$ clinical decision-making.

\section{Current Use:}

I currently use POCUS is some aspect of clinical care.

$67 \%$

\section{Barriers:}

Inadequate training in POCUS

$86 \%$

Inconvenient/Lack of access to ultrasound machine

$76 \%$

Inadequate time to incorporate POCUS into clinical practice

\section{Future Directions:}

I believe faculty would benefit from faculty development in POCUS.

$93 \%$

\section{program.}

With regard to current practice, a minority of hospitalists regularly incorporated POCUS into their clinical decisionmaking $(16 \%)$. The five most important indications for POCUS identified by respondents included evaluation of volume status (using the inferior vena cava), left ventricular function, peritoneal fluid, pericardial effusion and pleural effusion. Yet, only a fraction of them felt highly competent to perform and interpret these examinations $(5-47 \%$ depending on the indication, Figure 1). Moreover, a substantial proportion of hospitalists demonstrated knowledge deficits of the clinical applicability of POCUS. A fifth of respondents $(20 \%)$ omitted widely accepted uses of POCUS in hospital medicine (e.g. lung ultrasound in a hypoxic patient) and another fifth $(21 \%)$ endorsed POCUS for controversial, and generally non-recommended, indications (e.g. cardiac ultrasound to evaluate for aortic valvular disease).

The greatest barriers to POCUS use included inadequate training $(86 \%)$, inconvenient and/or lack of access to 


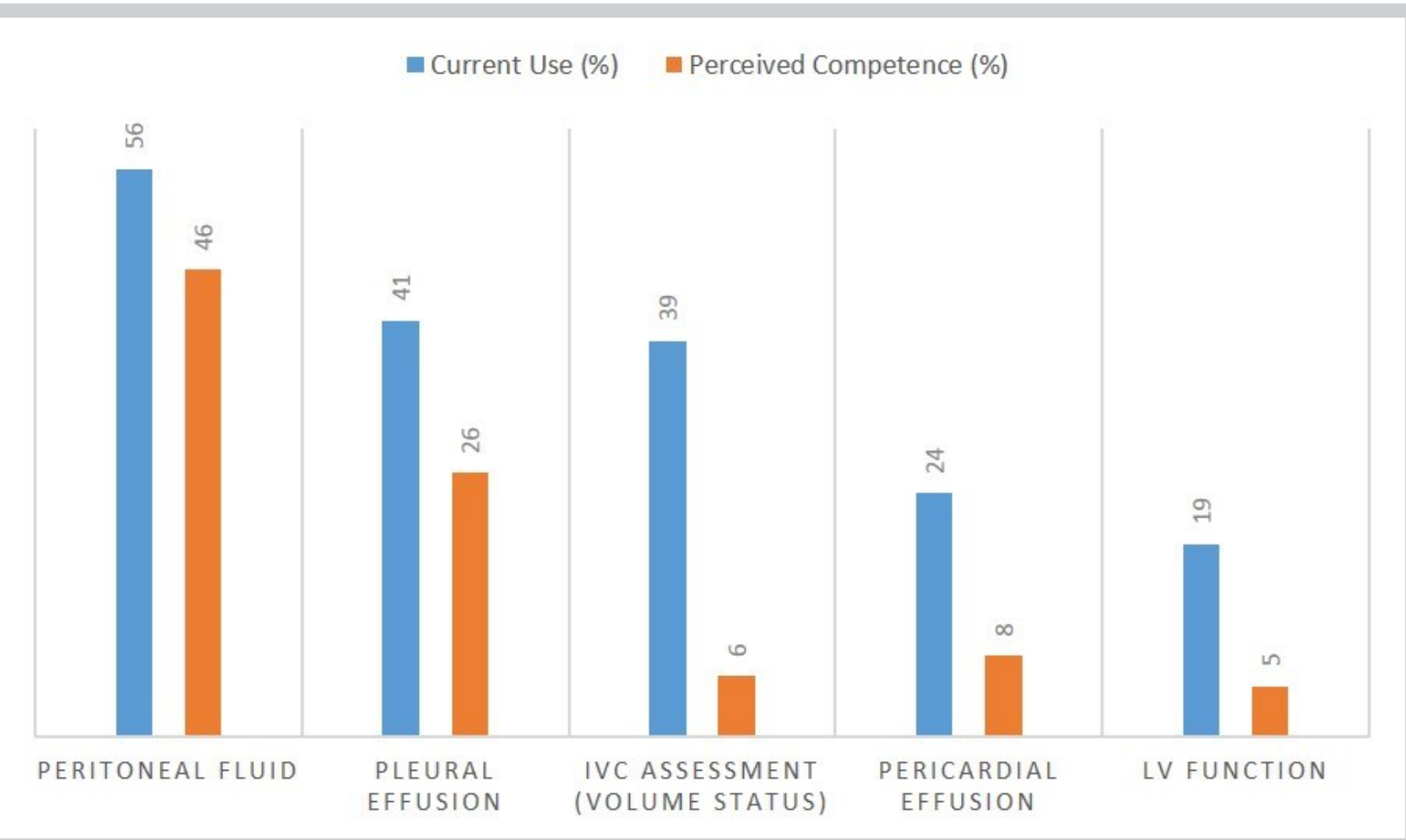

Figure 1. The Safety Gap Between Current Use and Perceived Competence. Survey respondents identified five domains of most frequent POCUS use in clinical practice: diagnostic evaluation of peritoneal fluid, pleural effusion, volume status (IVC), pericardial effusion, and LV function. However, hospitalists perceived competence in using POCUS for these five indications consistently fell below their reported use. We defined the gap between current use and perceived competence as the "safety gap" in faculty POCUS use.

ultrasound equipment (76\%), and inadequate time to incorporate it into their clinical workflow (52\%). If these barriers were addressed with proper training and access to equipment, most hospitalists could foresee themselves regularly incorporating POCUS into their clinical decisionmaking (73\%).

\section{Knowledge Test}

The response rate for the knowledge test was $57 \%$ (55/97). The overall average score was $41 \%$ with a wide range in test performance (11-67.5\%). Overall test scores and performance by I-AIM domain is summarized in Figure 2.

Test performance varied inversely based on level of experience with the most junior hospitalists (0-3 years since graduation from residency) scoring the highest $(56 \%)$, the most senior hospitalists ( $>10$ years since graduation from residency) scoring the lowest (22\%), and mid-career hospitalists (4-6 and 7-10 years from residency graduation) scoring in between $(37-43 \%, p=$ $<0.005)$.

The significant difference in total score was driven primarily by two I-AIM domains - image interpretation and clinical integration - where junior hospitalists performed the best $(54 \%$ and $58 \%$, respectively) and senior hospitalists performed the worst $(19 \%$ and $16.5 \%$, respectively; both $\mathrm{p}<0.005)$. Mid-career hospitalists performed consistently across all I-AIM domains (average scores ranging from $32-44 \%$ ). There was no significant difference in scores assessing understanding of POCUS indications or limitations ( $p=0.75$ and 0.21 , respectively), which appears to be driven by the relatively poor performance of all groups (range $33-44 \%$ and $25-46 \%$, respectively).

\section{Discussion}

The results of our surveys reveal three major gaps in POCUS utilization in this hospital medicine population. These gaps are primarily driven by a lack of training and is a ready target for intervention.

First, our needs assessment identified an "aspirational gap," which we have defined as the difference between the current and desired states of POCUS adoption. This gap represents hospitalists who believe in the potential for POCUS to improve clinical care, but struggle with the hurdles that create a high barrier to entry, such as constraints on time, training and equipment availability, and financial support from their institution. As a result, these hospitalists may never have the opportunity to engage with POCUS education in its current modes of 


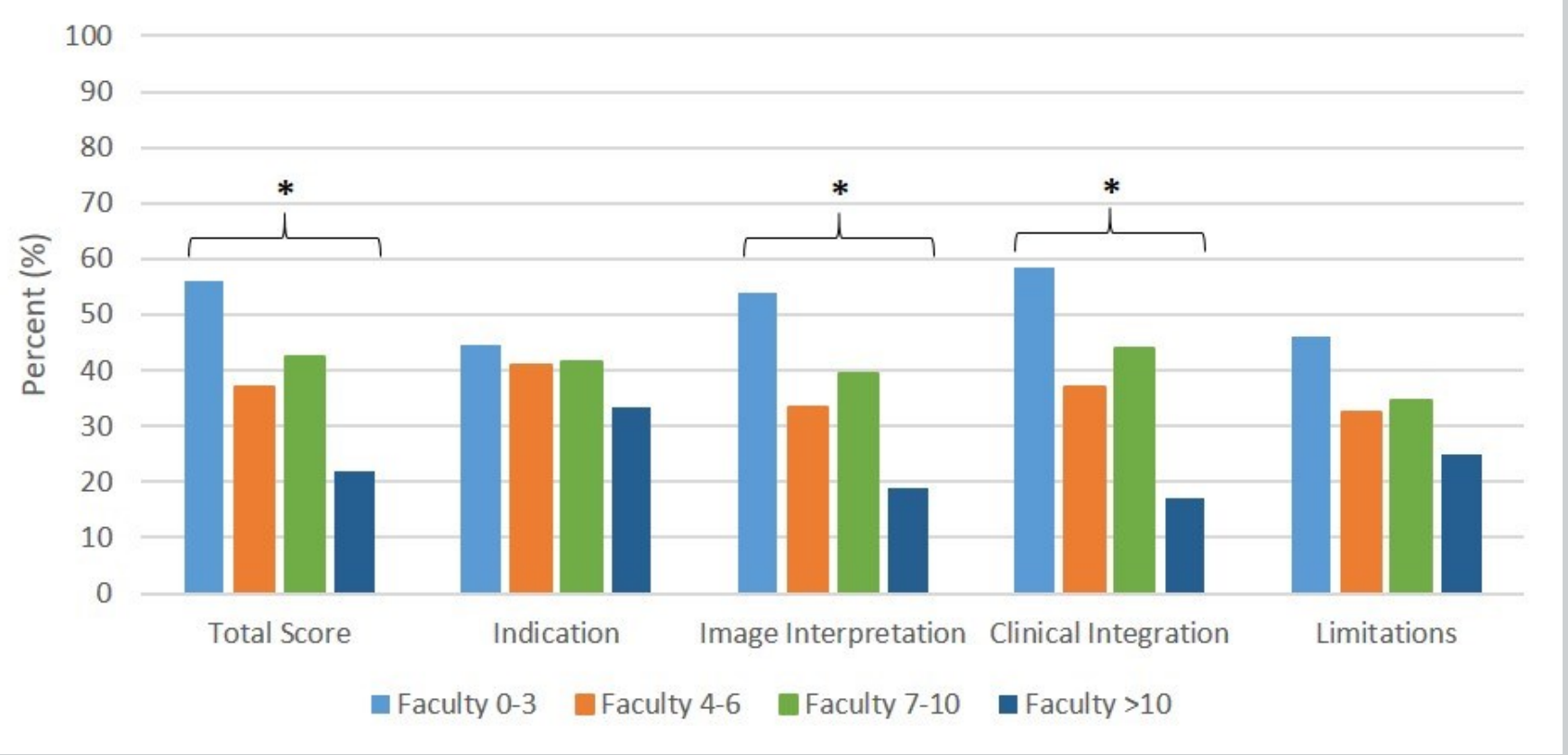

Figure 2. Knowledge Test Performance by Level of Hospitalist Experience. Hospitalist test scores varied significantly by level of experience ( $0-3$ years, $4-6$ years, $7-10$ years, and $>10$ years) in the domains of total score $(56,37$, $42.5,22 \%$ respectively; $p=0.0023)$, image interpretation $(54,33.5,39,19 \%$ respectively; $p=0.0015)$, and clinical integration $(58,37,44$, and $17 \%$ respectively; $p=0.0003)$. There was no significant difference between groups in the domains of POCUS indication $(44.5,41,41.5,33 \%$ respectively; $p=0.75)$ or understanding of limitations $(46$, $32,34.5,25 \%$ respectively; $p=0.21)$. Legend: ${ }^{*}=p<0.005$.

delivery. However, they still need to achieve a fundamental cognitive understanding of its basic principles, appropriate use, interpretation, and limitations in order to remain current given the growing body of physicians and trainees who have already integrated POCUS into their clinical practice.

Second, our needs assessment identified a "safety gap," which we have defined as the difference between current use and perceived competence in POCUS. This gap represents hospitalists who currently use POCUS without feeling confident in their abilities to accurately and reliably acquire and interpret ultrasound images with subsequent integration into their clinical decision-making. These hospitalists are more readily able to engage in POCUS education, but require additional training with emphasis on hands-on skill development and structured image review in order to promote high quality and safe clinical utilization of POCUS.

Third, our knowledge test revealed an overall deficit of understanding with respect to basic POCUS principles amongst all hospitalists, but particularly mid-career and senior hospitalists. These groups presumably had little-tono POCUS education during their residency training and are at highest risk of being eclipsed by more junior hospitalists and trainees as POCUS use expands. This knowledge deficit was most significant in the domains of image interpretation and clinical integration, where junior hospitalists demonstrated higher levels of proficiency. However, it is notable that no hospitalist group achieved an average score $>50 \%$ regarding POCUS indications or limitations, which is a concern for safety and appropriate POCUS use as this tool becomes more readily available.

Our survey had two major limitations. First, all data was collected from a single, academic center, which may limit its generalizability to all hospitalists. Secondly, our knowledge test questions were not previously validated. We believe that despite these limitations, our survey results reflect the current state of hospitalists with respect to their perspectives and knowledge of POCUS.

In order to address these gaps, we have developed a three-tiered POCUS curriculum that not only mandates cognitive competence for all hospitalists, but also offers expanded opportunities to engage up to their desired level of technical competence. Each tier is divided in order to achieve specific aims:

A/l hospitalists in basic knowledge and appropriate use of POCUS,

Some hospitalists in hands-on skill acquisition and image interpretation,

Few hospitalists in quality assurance and mastery of POCUS with resultant formal credentialing (Figure 3). 
Figure 3. Schematic of Curricular Design and Learning Objectives. This novel, tiered approach to POCUS education engages all hospitalists in basic POCUS knowledge and safety principles designed to aide them in resident supervision and collaboration with other inpatient providers (EM, ICU). Levels 2 offers voluntary training for hospitalists who want to develop the technical skill necessary to incorporate POCUS into clinical practice. Level 3 is structured as mentored portfolio development and assessment in order for hospitalists to achieve mastery and meet requirements for credentialing.

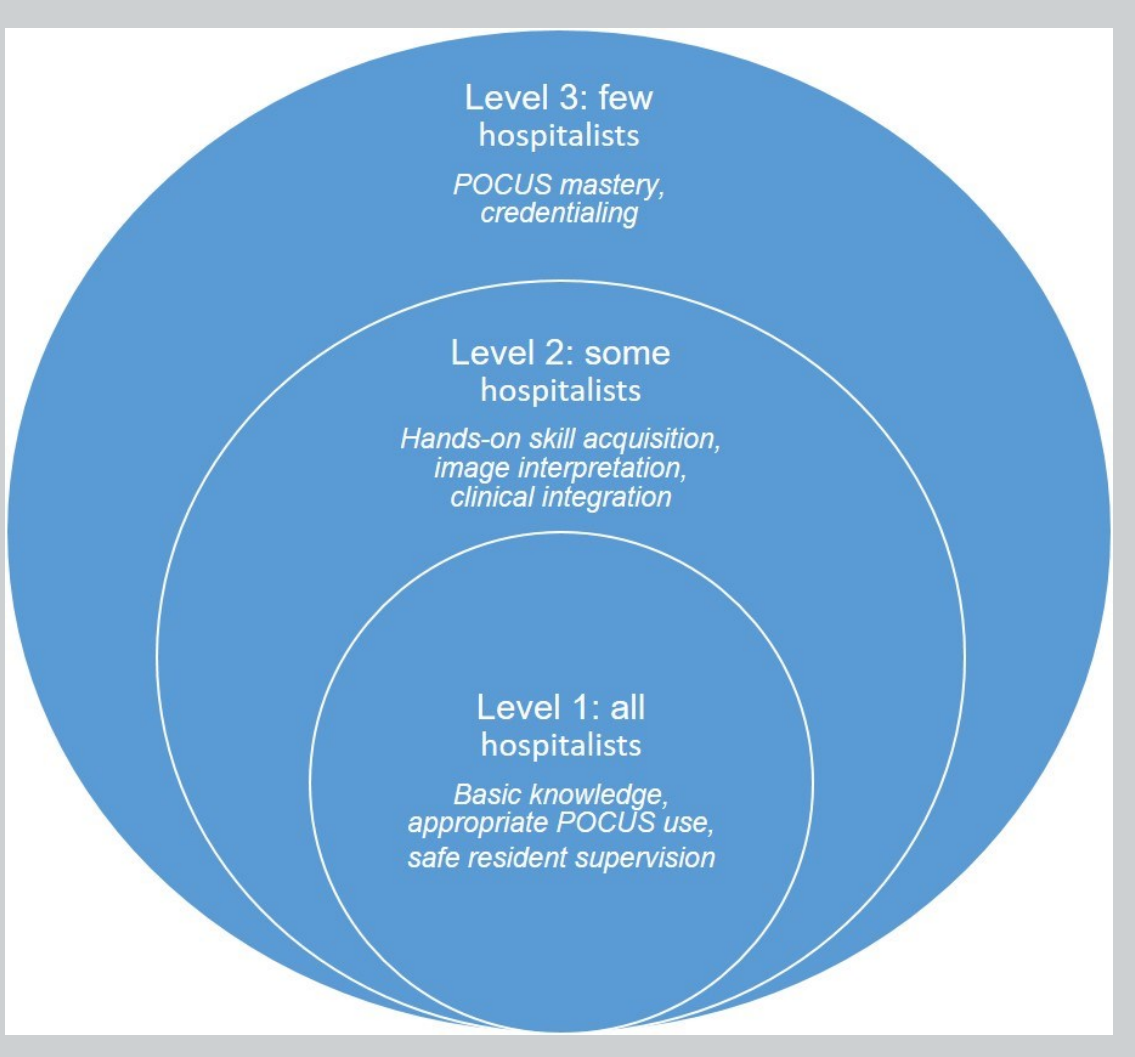

The purpose of tiered training is pragmatic: all hospitalists must achieve a baseline knowledge and familiarity with fundamental concepts, while interested hospitalists must be empowered with a pathway for technical competence as well as comprehensive mastery. This is critical for hospitalists who have resident physicians under their supervision and collaborating specialists in emergency medicine and critical care who use POCUS routinely, as well as those who wish to incorporate POCUS into their own clinical practice. We aim to achieve tiered training by offering core didactics in an accessible format with online modules for cognitive competence, supervised practice through hands-on sessions and scanning shifts for technical competence, and mentored portfolio building and longitudinal image review sessions for mastery. Lastly, by providing ongoing educational opportunities and mentorship, we hope to expand the cohort of advanced POCUS practitioners within our hospitalist group that not only use POCUS to deliver high quality care for our patients, but also teach it to all levels of learners.

\section{Conclusions}

Point-of-care ultrasound is an important skillset that will be essential to the practice of hospital medicine in the near future. A needs assessment of the present state of POCUS within our institution has identified both an "aspirational" and a "safety" gap between current and desired use of POCUS as well as current use and perceived competence in POCUS, respectively.
Furthermore, we identified significant knowledge gaps in all hospitalists, particularly amongst more senior hospitalists and in the domains of image interpretation and clinical integration. These gaps call for a novel, tiered curriculum that ensures a fundamental cognitive understanding of POCUS for all hospitalists, but also facilitates hands-on training for those who are committed to further skillset development ranging from technical competence for a limited set of indications to complete credentialing for the full spectrum of applications. We share a common concern that residents and other hospital-based providers will be using POCUS clinically to an extent that will eclipse the hospitalist's ability to safely supervise them or integrate their findings without expanding training in a pragmatic and thoughtful manner that accounts for current barriers to learning.

\section{Ethics Approval}

Institutional IRB at USCF reviewed and approved the project under exempt certification: (1) Research conducted in established or commonly accepted educational settings, involving normal educational practices, such as (i) research on regular and special education instructional strategies, or (ii) research on the effectiveness of or the comparison among instructional techniques, curricula, or classroom management methods. 


\section{References}

1. Lichtenstein DA, Mezière GA. Relevance of lung ultrasound in the diagnosis of acute respiratory failure: the BLUE protocol. Chest 2008; 134(1):117-25.

2. Perera P, Mailhot T, Riley D, Mandavia D. The RUSH exam: rapid ultrasound in SHock in the evaluation of the critically III, Emergency Medicine Clinics of North America 2010; 28(1): 29-56.

3. Perera P, Mailhot T, Riley D, Mandavia D. The RUSH exam: rapid ultrasound in SHock in the evaluation of the critically III, Ultrasound Clinics 2012; 7(2): 255-278.

4. Volpicelli G, Lamorte A, Tullio $M$, et al. Point-of-care multiorgan ultrasonography for the evaluation of undifferentiated hypotension in the emergency department. Intensive Care Med 2013; 39(7):1290-8.

5. Kajimoto K, Madeen K, Nakayama T, et al. Rapid evaluation by lungcardiac-inferior vena cava ( $\mathrm{LCl}$ ) integrated ultrasound for differentiating heart failure from pulmonary disease as the cause of acute dyspnea in the emergency setting. Cardiovasc Ultrasound 2012; 10(1): 49.

6. Yavaşi Ö, Ünlüer EE, Kayayurt K, et al. Monitoring the response to treatment of acute heart failure patients by ultrasonographic inferior vena cava collapsibility index. Am J Emerg Med 2014; 32(5): 403-7.

7. Corl KA, George NR, Romanoff J' et al. Inferior vena cava collapsibility detects fluid responsiveness among spontaneously breathing critically-ill patients. J Crit Care 2017; 41:130-137.

8. Subramaniam S, Bober J, Chao J, Zehtabchi S. Point-of-care Ultrasound for Diagnosis of Abscess in Skin and Soft Tissue Infections. Acad Emerg Med 2016; 23(11):1298-1306.

9. Howard ZD, Noble VE, Marill KA, et al. Bedside ultrasound maximizes patient satisfaction. J Em Med 2014. 46(1): 46-53.

10. Miller AH, Roth BA, Mills TJ, et al. Ultrasound Guidance versus the Landmark Technique for the Placement of Central Venous Catheters in the Emergency Department. Acad Emerg Med 2002. 9(8): 800-805.

11. Theodoro D, Blaivas $M$, Duggal $S$, et al. Real-time B-mode ultrasound in the ED saves time in the diagnosis of deep vein thrombosis (DVT). Am J Emerg Med 2004. 22(3): 197-200.

12. American Medical Association. House of Delegates. H-230.960 Privileging for ultrasound imaging. Available at: https://policysearch.amaassn.org/policyfinder/detail/Ultrasound\%20imaging?uri=\%2FAMADoc\% 2FHOD.xml-0-1591.xml. Accessed April 19, 2019

13. American College of Physicians. ACP Statement in Support of Pointof-Care Ultrasound in Internal Medicine. Available at: https:// www.acponline.org/meetings-courses/focused-topics/point-of-careultrasound-pocus-for-internal-medicine/acp-statement-in-support-of-point -of-care-ultrasound-in-internal-medicine. Accessed April 19, 2019.

14. Soni NJ, Schnobrich D, Mathews B, et al. Point-of-Care Ultrasound for Hospitalists: A Position Statement of the Society of Hospital Medicine. Published Online Only January 2, 2019. doi: 10.12788/jhm.3079

15. Mathews B, Zwank M. Hospital Medicine Point of Care Ultrasound Credentialing: An Example Protocol. J. Hosp. Med 2017; 9: 767-772.

16. Schnobrich DJ, Mathews BK, Trappey BE, Muthyala BK, Olson APJ. Entrusting internal medicine residents to use point of care ultrasound: Towards improved assessment and supervision. Med Teach 2018; 1-6.

17. Anstey JE, Jensen TP, Afshar N. Point-of-Care Ultrasound Needs Assessment, Curriculum Design, and Curriculum Assessment in a Large Academic Internal Medicine Residency Program. South Med J 2018; 111 (7): 444-448

18. Ma IWY, Arishenkoff S, Wiseman J, et al. Internal Medicine Point-ofCare Ultrasound Curriculum: Consensus Recommendations from the Canadian Internal Medicine Ultrasound (CIMUS) Group. J Gen Intern Med 2017; 32(9): 1052-7.

19. Mathews BK, Reierson K, Vuong K, et al. The Design and Evaluation of the Comprehensive Hospitalist Assessment and Mentorship with Portfolios (CHAMP) Ultrasound Program. J Hosp Med 2018; 13(8): 544550.

20. Bahner DP, Hughes D, Royall NA. I-AIM: A Novel Model for Teaching and Performing Focused Sonography. J Ultrasound Med 2012; 31: 295300 . 